Revue d'histoire de l'Amérique française

REVUE D.HISTOIRE DE L'AMÉRIQUE FRANÇAISE

\title{
En filigrane des récits du père Louis Hennepin " trous noirs " de l'exportation louisianaise, 1679-1681
}

\section{Catherine Broué}

Volume 53, numéro 3, hiver 2000

URI : https://id.erudit.org/iderudit/005344ar

DOI : https://doi.org/10.7202/005344ar

Aller au sommaire du numéro

\section{Éditeur(s)}

Institut d'histoire de l'Amérique française

\section{ISSN}

0035-2357 (imprimé)

1492-1383 (numérique)

Découvrir la revue

\section{Citer cet article}

Broué, C. (2000). En filigrane des récits du père Louis Hennepin " trous noirs " de l'exportation louisianaise, 1679-1681. Revue d'histoire de l'Amérique française, 53(3), 339-366. https://doi.org/10.7202/005344ar

\section{Résumé de l'article}

La Description de la Louisiane (1683) et la Nouvelle Decouverte (1697) du père Louis Hennepin, missionnaire récollet ayant participé à l'exploration du Mississippi, dirigée par l'explorateur Cavelier de La Salle, de 1678 à 1681 comportent des contradictions et des vides narratifs que les historiens ont souvent considérés comme des erreurs et des manques, voire des mensonges rendant inutilisables ces relations pourtant riches en renseignements de toutes sortes. Le propos de cet article est de montrer que ces contradictions et ces silences pourraient bien être délibérés, issus d'une rhétorique habile visant à occulter certains agissements du récollet ou de son supérieur La Salle. Une analyse serrée de ces récits et l'examen d'autres témoignages (dont certaines lettres de La Salle) permettent d'esquisser une hypothèse quant à la destination du canot envoyé par La Salle au printemps 1680. 


\title{
EN FILIGRANE DES RÉCITS DU PÈRE LOUIS HENNEPIN «TROUS NOIRS $»$ DE L'EXPLORATION LOUISIANAISE, 1679-1681
}

\author{
CATHERINE BROUÉ \\ Département d'histoire \\ Université Laval
}

\section{RÉSUMÉ}

La Description de la Louisiane (1683) et la Nouvelle Decouverte (1697) du père Louis Hennepin, missionnaire récollet ayant participé à l'exploration du Mississippi, dirigée par l'explorateur Cavelier de La Salle, de 1678 à 1681 comportent des contradictions et des vides narratifs que les historiens ont souvent considérés comme des erreurs et des manques, voire des mensonges rendant inutilisables ces relations pourtant riches en renseignements de toutes sortes. Le propos de cet article est de montrer que ces contradictions et ces silences pourraient bien être délibérés, issus d'une rhétorique habile visant à occulter certains agissements du récollet ou de son supérieur La Salle. Une analyse serrée de ces récits et l'examen d'autres témoignages (dont certaines lettres de La Salle) permettent d'esquisser une hypothèse quant à la destination du canot envoyé par La Salle au printemps 1680.

\section{ABSTRACT}

By virtue of their internal contradictions, and a number of narrative «holes», Louis Hennepin's Description de la Louisiane (1683) and his Nouvelle Decouverte (1697), which relate the Recollect missionary's participation in the Mississippi-Valley explorations organized by Cavelier de La Salle from 1678 to 1681, are often considered to be unreliable sources, riddled with mistakes or even untruths. This paper argues instead that the narratives' contradictions and silences may have been deliberate rhetorical devices aimed at masking some of the missionary's or the explorer's more dubious actions. In particular, when the two relations are examined in conjunction with other sources, such as La Salle's letters, they suggest new hypotheses about the canoe sent by La Salle in 1689. More generally, this close literary analysis of Hennepin's texts invites historians to reconsider and revisit what amount to rich, information-laden sources. 
Quoy que la découverte fût déja avancée de quatre à cinq cens lieuës dans la Loüisiane, depuis le

Fort de Frontenac, jusques à celuy de Creve-cœur;

l'on ne doit considerer cette grande route, que comme un Prelude \& un préparatif à de plus vastes entreprises, \& une entrée que l'on se faisoit dans des païs beaucoup plus avantageux'.

J'ay creu qu'il estoit à propos de vous faire le narré des aventures de ce canot, parce que

je ne doute pas qu'on en parle, et si vous en souhaitez conférer avec le Père Louis Hempin, Recollect, qui est repassé en France, il faut un peu le connoistre, car il ne manquera pas d'exagérer toutes choses: c'est son caractère ${ }^{2}$.

Je me trouvay enveloppé par la conjoncture du temps dans de grandes \& de facheuses affaires tant du côté du Sieur de la Salle, que de celui de ces deux hommes, que j'avois avec moy, \& qui devoient m'accompagner dans mon Voiage $e^{3}$.

Le 12 mai 1678, René-Robert Cavelier de La Salle obtient de Louis XIV la permission de découvrir la partie occidentale de la Nouvelle-France ${ }^{4}$. De retour à Québec à la mi-septembre $1678^{5}$, il envoie aussitôt un premier groupe de travailleurs et d'artisans à l'extrémité ouest du lac Ontario, afin qu'ils y construisent un entrepôt et entreprennent la fabrication d'un navire avec lequel il compte traver-

1. C. Leclercq, Premier etablissement de la Foy dans la Nouvelle-France (Paris, Amable Auroy, 1691), II : 161-162. Le texte avec lequel j'ai travaillé a été établi par R. Ouellet sur l'exemplaire du Musée de l'Amérique française.

2. La Salle, lettre du 22 août 168[1], dans P. Margry, Découvertes et établissements des Français dans l'ouest et dans le sud de l'Amérique septentrionale, 1614-1698, Mémoires et documents inédits (Paris, Maisonneuve et Cie, 1879-1888), II : 259. Cette lettre est conservée à la Bibliothèque nationale de France, Fonds Clairambault, 1016, f. 65 r-84. La date assignée par P. Margry dans sa transcription est le 22 août 1682. Sur la controverse entourant cette date, voir H. Lemay, Bibliographie du Père Louis Hennepin, récollet. Les Pièces documentaires (Montréal, 1937), 27 ainsi que J. Delanglez, «A Calendar of La Salle's Travels, 1643-1683», Mid-America, 22 (1940): 292 et Hennepin's Description of Louisiana. A Critical Essay (Chicago, Institute of Jesuit History, 1941), 84. Bien que, pour des raisons d'ordre pratique, je me serve ici de la transcription de P. Margry, je ferai référence à cette lettre en mentionnant la date du 22 août $168[1]$.

3. Louis Hennepin, Nouvelle Decouverte d'un tres grand Pays Situé dans l'Amerique, entre Le Nouveau Mexique, et La Mer Glaciale, Avec les Cartes, \& les Figures necessaires, \& de plus l'Histoire Naturelle \& Morale, \& les avantages qu'on peut en tirer par l'établissement des Colonies (Utrecht, chez Guillaume Broedelet, 1697), 250. Dorénavant, je ferai mention de cet ouvrage sous le titre abrégé de Nouvelle Découverte.

4. Permission au $\mathrm{Sr}$ de la Salle de descouvrir la partie occidentalle de la nouvelle france, Archives nationales de Paris, Colonies, F3-5, f. 41-42.

5. Le 15 septembre d'après J. Delanglez, loc. cit., 290. 
ser les Grands Lacs. Louis Hennepin, missionnaire récollet, assure la conduite spirituelle du groupe ${ }^{6}$. Un an plus tard, toute l'équipe dirigée par La Salle se trouve rassemblée sur la rivière Illinois où l'explorateur fait entreprendre la construction d'un second navire et d'un établissement fortifié baptisé Crèvecœur. C'est de ce fort qu'à la demande de La Salle, le soir du 29 février 1680, Louis Hennepin part à nouveau en éclaireur pour accompagner deux engagés, Antoine Auguelle et Michel Accault, dans un audacieux périple en canot d'écorce vers le Mississippi? ${ }^{7}$. Au bord du grand fleuve, le 11 avril 1680 , le canot du petit groupe est aperçu par une armée de guerriers dakotas qui décident d'amener ces hommes dans leur pays ${ }^{8}$. Cette rencontre imprévue met un terme à la participation du récollet à l'entreprise de La Salle : après un séjour de quelques mois parmi les Sioux Santees, Hennepin hiverne à Michilimackinac, puis repart à Québec dès le printemps sans avoir revu l'explorateur'. Il repasse en France peu après ${ }^{10}$. Là, il rédige la Description de la Louisiane ${ }^{11}$, qui couvre les trois dernières années de son séjour en Nouvelle-France. De son périple en canot solitaire, son ouvrage rapporte, essentiellement, l'aventure siouse des trois hommes. Évasif quant à la direction prise au sortir de la rivière Illinois, le missionnaire résume par une ellipse un mois de voyage. Quinze ans plus tard, ce vide narratif lui permet-

6. La Relation des decouvertes et des Voyages du Sieur de la Salle, seigneur et gouverneur du Fort de Frontenac, au dela des grands Lacs de la Nouvelle France faits par l'Ordre de Monseigneur Colbert 1679. 80. et 81, présentée à la cour en 1682, reconnaît à Hennepin un rôle de premier plan lors de cette phase préliminaire de l'exploration: «Au Commencement de l'année suivante 1679. Il envoya ses Charpentiers à Niagara avec d'autres Gens sous la conduitte du Sr de la Motte et du Pere Louis Henpin Missionaire Recolet pour y faire un Fort et construire une Barque a l'entrée du Lac Erié» (d'après la copie conservée aux Archives nationales de France, Marine, 3JJ271, f. $273 \mathrm{v}^{\circ}$ ). Les citations de cette Relation seront toutes extraites, dans cet article, du document d'archives précité.

7. La Salle consigne ce départ dans sa lettre du 29 septembre 1680, éditée par I. Paquet, Édition critique d'une lettre de Cavelier de La Salle à son associé Thouret, mémoire de maîtrise, Université Laval, 1997, 174.

8. La Salle rapporte ces événements dans sa lettre du 22 août 168[1], dans P. Margry, op. cit., 212-262.

9. C'est du moins ce que laissent croire les ouvrages de Louis Hennepin et la lettre de la Salle du 22 août 168[1], dans P. Margry, op. cit., 259-260.

10. A. Louant se base sur les termes d'une lettre de Hennepin à Louis Errembault pour avancer comme date de ce retour le début de décembre 1682, dans Le Cas du père Louis Hennepin, récollet, Missionnaire de la Louisiane 1626-170.? ou Histoire d'une vengeance, Annales du Cercle royal d'histoire et d'archéologie d'Ath et de la région et Musées Athois, 47 (1978-1979): 65. Sur le séjour parisien du récollet, voir également H. Lemay, «Le Père Louis Hennepin récollet à Paris, $1682 »$, Nos cahiers, 2,2 (1938): 105-140.

11. Description de la Louisiane, Nouvellement Decouverte au Sud'Oüest de la Nouvelle France, par ordre du Roy. Avec la Carte du Pays: Les Moeurs \& la Maniere de vivre des Sauvages. Dediée a sa Majesté Par le R. P. Loüis Hennepin, Missionnaire Recollet \& Notaire Apostolique (Paris, Amable Auroy, 1683 et 1688), 309 p. Je ferai dorénavant mention de cet ouvrage sous le titre abrégé de Description de la Louisiane. 
tra, dans un second ouvrage ${ }^{12}$, de revendiquer la primeur de la découverte de l'embouchure du grand fleuve que les trois hommes auraient descendu deux ans avant Cavelier de La Salle, au cours du mois précédant leur rencontre avec les Dakotas ${ }^{13}$. Plusieurs générations d'historiens ${ }^{14}$ ont contesté cette revendication en cherchant à répondre à cette interrogation que les récits du père Hennepin leur semblaient susciter: le missionnaire a-t-il vraiment descendu le Mississippi ?

Tout autre avait été la question de l'Avare de Molière: "mais qu'allait-il donc faire dans cette galère »? La rhétorique narrative du missionnaire ${ }^{15}$ camoufle si bien l'enjeu de cette expédition en canot que personne, jusqu'ici, n'a véritablement cherché à comprendre quelle avait été la mission des trois hommes ${ }^{16}$. Les contradictions de cet épisode et le vide narratif entourant près d'un mois de navigation en pays inconnu m'ont amenée à m'interroger sur la nature de ce référent manifestement embarrassant. Dans la trame textuelle du récit du père Hennepin et sous les explications parfois confuses fournies par La Salle à ce sujet se profilent quelques éléments de réponse à cette question prosaïque: qu'allait-il donc faire dans ce canot?

Certes, il est hasardeux de vouloir retrouver, dans un ouvrage si controversé depuis sa parution et dont la recherche historique semble unanime à décrier les exagérations et les invraisemblances ${ }^{17}$, des traces

12. Nouvelle Découverte, 1697.

13. Ibid., 248-314.

14. Citons seulement J. G. Shea, G. Gravier, M. De Villiers, J. Delanglez, H. Lemay et, plus récemment, A. Louant.

15. J'ai déjà proposé une analyse rhétorique de la Description de la Louisiane dans «Les enjeux de l'écriture», Missionnaires et écrivains récollets à la fin du XVII siècle, Journées d'études du CELAT, 13 et 14 juin 1996.

16. Intrigué par l'absence de tout détail narratif ou descriptif entre le 12 mars et le 12 avril 1680, J. G. Shea suggère deux explications: Hennepin aurait descendu le Mississippi, ou aurait agi d'une quelconque façon contre les ordres de La Salle (Discovery and Exploration of the Mississippi Valley: with the Original Narratives of Marquette, Allouez, Membre, Hennepin and Anastase Douay), J. S. Redfield (1852), 106. J. Delanglez semble également se poser la question: si Hennepin avait pensé trouver la route de la Chine et du Japon en 1682, pourquoi n'en aurait-il pas parlé en haut lieu à Paris? fait-il mine de s'interroger en 1947 («A Mirage : The Sea of The West», Revue d'histoire de l'Amérique française, 1,2 (septembre 1947): 372). Malheureusement, sa question n'en est pas une: elle constitue un argument à sa démonstration voulant que ni La Salle ni Hennepin n'aient jamais tenté de pousser leurs découvertes vers l'ouest. Au contraire, G. L. Nute croit qu'Hennepin désirait bel et bien se rendre chez les Sioux et avance que cette expédition des trois hommes n'était pas sans rapport avec la volonté de La Salle de découvrir une route vers la mer de l'ouest; Caesars of the Wilderness. Médard Chouart, Sieur des Groseilliers and Pierre Esprit Radisson, 1618-1710 (New York/London, D. Appleton/ Century Company Inc., 1943), 182.

17. Par exemple, dans sa revue au demeurant minutieuse des sources sur l'expédition de La Salle de 1682, P. Galloway signale qu'elle n'a pas cru bon d'intégrer l'ouvrage d'Hennepin [elle ne précise pas lequel. Il s'agit sans doute du Nouveau Voyage d'un Pais plus grand que l'Europe Avec les relections des entreprises du Sieur de la Salle, sur les Mines de St. Barbe [...] 
d'un passé au demeurant déjà minutieusement épluché. Toutefois, la perspective littéraire avec laquelle j'ai jusqu'ici étudié l'œuvre de Louis Hennepin ${ }^{18}$ dévoile une dimension insoupçonnée de ces textes: celle du sous-entendu et du non-dit. Loin d'être un obstacle à la compréhension, les incohérences, les contradictions et les silences de la Description de la Louisiane constitueront donc, dans le présent essai, la trame fondamentale permettant de décrypter, sous les conventions ou les intentions scripturales, une vérité travestie. Incidemment, le «filigrane » du texte de Hennepin révèle certaines ombres des écrits de La Salle et pourrait jeter une lumière nouvelle sur un pan important de l'histoire de la Nouvelle-France. Précisons toutefois que le propos de cet article n'est pas d'établir une nouvelle vérité historique ou ethnohistorique, mais de proposer une lecture renouvelée des récits de Louis Hennepin et, surtout, des silences et des contradictions qu'ils mettent en scène. Cette lecture découvre des sens cachés, glissés, volontairement ou non, par l'auteur dans ses récits, et qui subvertissent les textes donnés à lire ${ }^{19}$.

\section{UN RÉCIT BROUILLÉ}

En février 1680, La Salle et son groupe s'affairent à construire un établissement fortifié ainsi qu'un bateau sur le bord de la rivière Illinois, en aval du lac Pimiteoui. Depuis la mi-janvier, le père Zénobe Membré, l'un des trois récollets accompagnant le groupe, passe ses

(Utrecht, Antoine Schouten, 1698), 389 p.] à cet inventaire: «the Hennepin account, now universally accepted as entirely spurious, has been omitted», "Sources for the La Salle Expedition of $1682 »$, dans P. Galloway, dir., La Salle and His Legacy. Frenchmen and Indians in the Lower Mississippi Valley (Jackson, University Press of Mississippi, 1982), 22.

18. Avant moi, plusieurs chercheurs ont su exploiter la richesse littéraire des ouvrages d'Hennepin. Ainsi, N. Doiron, P. Berthiaume, R. Ouellet ont puisé dans ses récits des exemples à l'appui de leurs avancées théoriques sur la relation de voyage; par exemple, N. Doiron, «De l'épreuve de l'espace au lieu du texte. Le récit de voyage comme genre», Voyages, récits et imaginaire. Actes de Montréal édités par Bernard Beugnot (Paris, Biblio 17), 15-31; P. Berthiaume, L'aventure américaine au XVIII siècle. Du voyage à l'écriture (Ottawa, Presses de l'Université d'Ottawa, 1990), 487 p.; R. Ouellet et C. Rigault, «Sur la Nouvelle-France : documents et questionnements », Études littéraires, 10,1-2 (avril-août 1977): 9-18. L'article d'Hélène Vachon, «L'implicite comme langage publicitaire — Étude de la syntaxe temporelle dans la Description de la Louisiane», Études littéraires, 10,1-2 (avril-août 1977): 175-194, marque également une date importante pour l'analyse littéraire de l'œuvre de Hennepin.

19. Dans le cadre d'un doctorat en littérature française consistant en l'édition critique de deux récits du récollet Louis Hennepin, qui a participé de 1678 à 1681 à l'exploration de la Louisiane par René-Robert Cavelier de La Salle, j'ai été amenée à effectuer une analyse narrative de ces récits dont je propose ici une petite partie, susceptible d'intéresser la recherche. Pour en savoir davantage sur le contexte historique, approfondir l'analyse littéraire ou consulter l'édition critique des deux œuvres étudiées, voir C. Broué, Édition critique de la Description de la Louisiane et de la Nouvelle Découverte du père Louis Hennepin, thèse de doctorat, Université Laval, 1999, 654 p. 
journées dans le camp hivernal des Illinois, établi non loin de là, et prévoit suivre ces Amérindiens jusqu'à leur village d'été quand ces derniers commenceront à y retourner. Le père Gabriel de la Ribourde, supérieur de la mission, décide «de rester au Fort avec le sieur de Tonty et les travaillants ${ }^{20} »$. Louis Hennepin a résolu «d'aller avec deux hommes dans des pays inconnus où on est à tout moment dans un grand danger de sa vie ${ }^{21} »$. Cette répartition des tâches ne s'est pas faite sans discussions. En effet, souligne le récit, le père Zénobe n'est pas très enthousiaste à l'idée de rester chez les Illinois, mais préfère encore les «manières importunes» de cette tribu ${ }^{22}$ aux dangers qui guettent le voyageur.

Nous voyans trois Missionnaires Recolets avec le peu de François que nous avions au Fort de Crevecoeur, \& n'ayant plus de vin pour celebrer la Messe, [...] le Pere Zenoble [...] commençoit à s'ennuyer, ayant peine à se faire à la maniere importune des Suavages, avec lesquels il demeuroit, nous en parlâmes au Sieur de la Salle [...] je m'offris à prendre sa Mission, pourveu qu'il voulut aller à ma Place aux Nations ulterieures, dont nous n'avions point encore de connoissance, que celle que les Sauvages nous en avoient donnée, qui n'étoit que superficielle, c'est ce qui donna à penser au Pere, lequel aima mieux rester avec les Islinois, dont il avoit connoissance, que de s'exposer d'aller chez des peuples inconnus ${ }^{23}$.

C'est, narrativement parlant, après que le missionnaire ait décidé de partir que La Salle lui demande de se joindre aux deux hommes. La succession surprenante de ces deux événements (Résolution d'Hennepin/Proposition de La Salle) semble révéler qu'Hennepin s'était offert à un autre voyage avant que La Salle ne lui propose une route à «découvrir». Entre les deux protagonistes, un conflit sousjacent se laisse percevoir, que confirme cette citation tirée du Premier établissement de la Foy:

20. Relation attribuée à Membré dans C. Leclercq, op. cit., 170.

21. Description de la Louisiane, 172-173.

22. Le récit attribué à Zénobe Membré dans le Premier établissement de la Foy souligne également ce découragement du père: «il y a tant d'éloignement de la foi parmy ces Barbares, un esprit si brutal \& si bouché, des mœurs si corrompues et opposées au Christianisme, qu'il faudra bien du temps pour esperer quelque fruit [...]. Je fis aussi un voyage aux Myamis pour apprendre quelque chose de leurs dispositions, de là j'allais visiter d'autres Villages des Illinois, tout cela sans beaucoup de succés, ne trouvant que des sujets de chagrins, sur l'état déplorable \& l'aveuglement de ces Nations, je ne sçaurois exprimer tout ce qui en est.» C. Leclercq, op. cit., 180-181.

23. Description de la Louisiane, 182-183. 
Comme l'on avoit destiné le Pere Loüis, \& qu'il s'estoit offert d'avancer la découverte du côté Nord, en remontant le Fleuve, le sieur de la Salle s'étant reservé de la continuer en Canot, en descendant jusques où l'on pourroit trouver la Mer; le Pere Loüis partit en canot du Fort de Creve-coeur ${ }^{24}[\ldots]$

Ce passage fait bien une distinction entre ce qu' «on» avait décidé pour le père Louis et ce que ce dernier avait lui-même résolu (même si la phrase présente comme similaires les deux décisions) et oppose le couple Demande à Hennepin/Proposition d'Hennepin au fait que La Salle se réservait la descente du Mississippi. La découverte de Louis Hennepin ne serait donc pas celle que le missionnaire espérait ${ }^{25}$, et cela pourrait suffire à expliquer ses prétentions subséquentes ${ }^{26}$.

Mais qu'en est-il donc du voyage commandé? La Salle aurait offert au récollet une mission bien particulière:

Et avant que de retourner au Fort de Frontenac pour aller querir du renfort, des Cables et des Agretz pour la derniere Barque qu'il laissa faite jusques au Cordon; il me pria de vouloir prendre la peine d'aller découvrir par avance la route qu'il devoit tenir jusques à la Riviere Colbert, à son retour de Canada ${ }^{27}$.

Que faut-il comprendre de cette demande? S'agit-il seulement d'achever la descente de la rivière Illinois jusqu'au Mississippi? La suite du récit montre clairement que l'expédition des trois hommes ne doit pas s'arrêter à l'embouchure de l'Illinois. Que cache alors cette formulation sybilline prêtée à l'explorateur? Un enjeu manifestement important, que le narrateur signale au lecteur par une objection surprenante: un abcès à la bouche nécessiterait son retour en Canada. Il faut

24. Relation attribuée à Zénobe Membré dans C. Leclercq, op. cit., 160-161.

25. La rancœur du missionnaire se laissera mieux percevoir dans ses ouvrages subséquents. Voir C. Broué, «Récits d'un voyage ou voyage d'un récit: de la Description de la Louisiane à la Nouvelle Découverte du père Louis Hennepin », communication présentée au $49^{\circ}$ congrès de l'Institut d'histoire de l'Amérique française, octobre 1994.

26. Armand Louant va jusqu'à avancer qu'en 1682, Hennepin aurait présenté à la cour, avec le récit qui constituera la Description de la Louisiane, un deuxième cahier contenant notamment le récit de la descente du Mississippi, loc. cit., 76-84. De fait, le travail de «mise au flou » de la première partie du voyage du récollet sur le Mississippi, dans la Description de la Louisiane, préfigure le récit de la descente du Mississippi ajouté dans la Nouvelle Découverte. Véritable ou mensongère, cette «révélation» du second ouvrage d'Hennepin fait écho au silence du premier. On pourrait dès lors la considérer à son tour comme une même allégorie d'un mystère encore entier jusqu'ici ou, plus simplement, comme une sorte d'alibi à une accusation dont les échos ne nous seraient pas parvenus. Ainsi, les modifications apportées par Hennepin à son premier récit dans la Nouvelle Découverte, peuvent également servir à révéler un sens resté caché dans la Description de la Louisiane.

27. Description de la Louisiane, 184. 


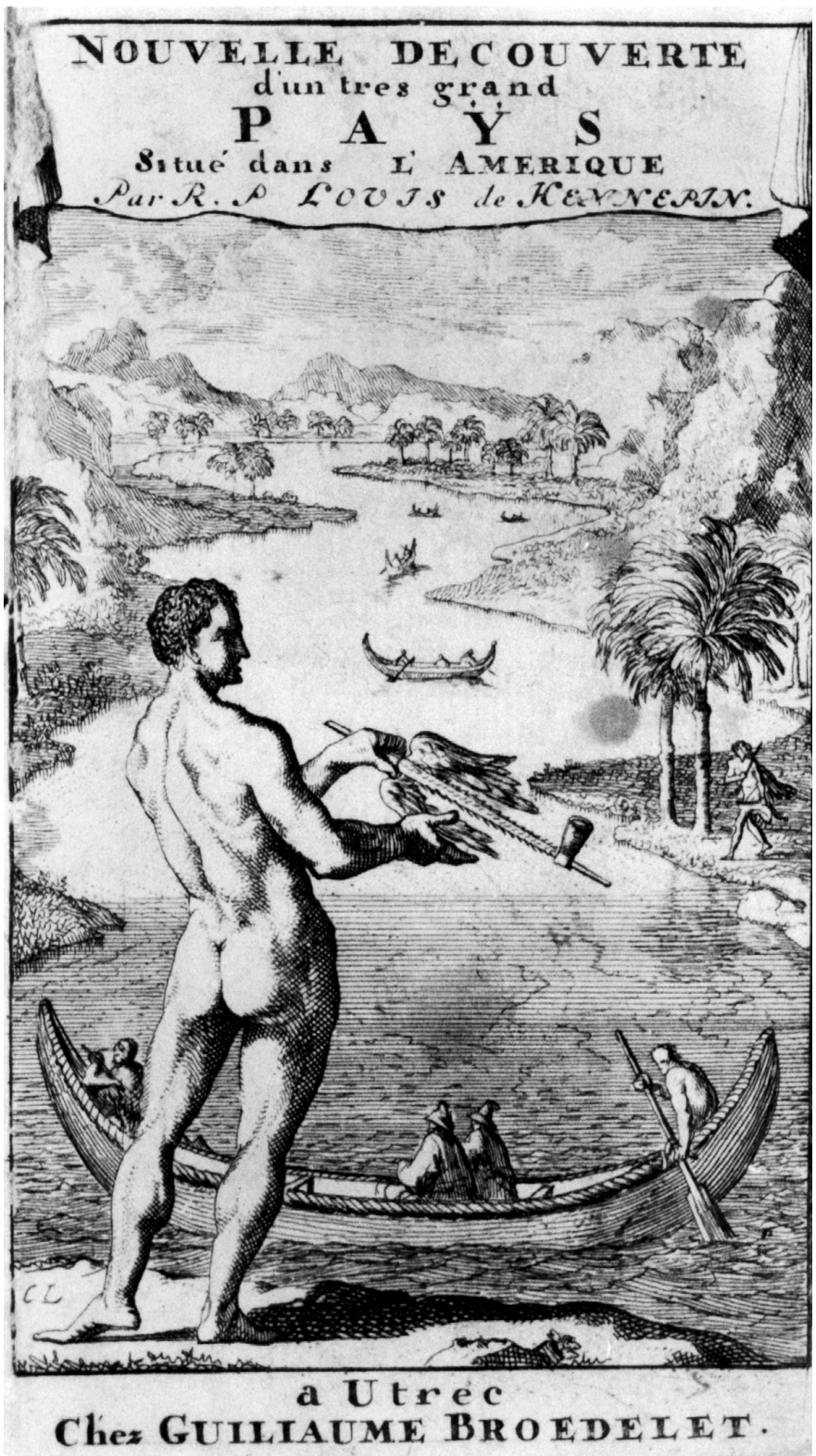

Amérindien portant un calumet

Source: Frontispice de la Nouvelle Decouverte d'un Tres grand Pays Situé dans l'Amérique, 1697, gravure extraite de l'exemplaire de la Bibliothèque nationale, RES AF 95. 
la menace d'éventuelles sanctions ecclésiastiques et l'intervention de son supérieur pour que le récollet obtempère.

comme j'avois un abscez à la bouche qui suppuroit continuellement \& qui me duroit depuis un an \& demy, je luy témoignay ma repugnance, $\&$ luy dis que j'avois besoin de retourner en Canada pour me faire penser, il me répondit que si je refusois ce voyage, qu'il écriroit à mes Superieurs que je serois la cause du peu de succés de nos nouvelles Missions ${ }^{28}$.

On est en droit de s'étonner de cette incommodité imprévue: le lecteur n'en a pas encore entendu parler, et l'abcès ne se manifestera à aucun autre moment du récit. Son caractère insolite a justement éveillé mon attention: que cache l'embarras soudain du missionnaire? Quel abcès pourrait empêcher le bouillant Hennepin de prendre encore une fois les devants de l'exploration? La réponse pourrait provenir d'une lecture non pas littérale, mais allégorique de ce passage ${ }^{29}$. Datée par le narrateur, la naissance de l'abcès remonte en effet à l'automne de 1678, au moment où Hennepin commence à participer à l'expédition de La Salle. L'endroit de cet abcès, la bouche, suggère une parole ravalée, ou un mensonge, chargeant la conscience du missionnaire qui sentirait alors la nécessité de «se faire penser ${ }^{30}$ ». La «répugnance» témoignée semble liée à son honneur d'ecclésiastique: plus que les menaces, c'est l'exemple du père Gabriel qui incite finalement Louis Hennepin à «passer outre» :

Le Reverend Pere Gabriel de la Ribourde qui avoit esté mon Pere maître du Noviciat, me pria de passer outre [...], considerant [...] que ce vieillard estoit l'unique enfant mâle et heritier de la maison de son pere, je m'offris à faire ce voyage ${ }^{31}$.

Sur le mode allégorique, Hennepin s'insurgerait contre certaines pratiques de La Salle, mais accepterait finalement de les couvrir pour le bien de la Mission. Le remaniement du même passage dans la Nouvelle Découverte, même s'il semble atténuer la portée de cet abcès, nous conforte dans cette lecture:

28. Ibid., 184-185.

29. C. Broué, «Les enjeux de l'écriture», CÉLAT, 13 et 14 juin 1996.

30. Les deux verbes «penser» et «panser» sont issus du même verbe latin pensare. Au XVII ${ }^{e}$ siècle, la graphie "penser» est encore couramment utilisée dans le sens médical de l'homonyme. Cet usage disparaît toutefois au XVIII ${ }^{\mathrm{e}}$ siècle; O. Bloch et N. Von Wartburg, Dictionnaire étymologique de la langue française (Paris, Presses universitaires de France, 1986), $7^{e}$ édition. L'ambiguïté de l'orthographe pourrait bien être ici délibérée.

31. Description de la Louisiane, 186-187. 
J'avois un Abcés à la bouche, qui suppuroit tous les jours depuis un An \& demi, quoi que sans puanteur. Je lui temoignay la repugnance, que j'avois à faire le Voiage, dont il s'agissoit, $[$ [...] Le bon Pere Gabriel de la Ribourde, qui avoit été mon Pere Maître de Noviciat dans nôtre Convent de Bethune au pays d'Artois, me pria de passer outre [...] Considerant donc, que ce bon Vieillard [...] étant l'unique heritier d'une Maison noble de Bourgogne, il avoit bien voulu sacrifier tout cela à l'honneur de la Mission, j'entrepris ce dangereux voiage avec une entière assurance $^{32}$.

Par ailleurs, la reprise de ce même mot de «répugnance », dans la Description de la Louisiane, lorsque le missionnaire craint pour sa vie, souligne par contrecoup l'intensité dramatique de l'épisode de l'abcès.

j'estois d'avis de me laisser tuer sans aucune resistance, puis que je leur allois annoncer un Dieu qui a esté accusé faussement, condamné injustement, \& crucifié cruellement, sans qu'il ait jamais témoigné la moindre repugnance à ceux qui le faisoient mourir ${ }^{33}$.

Accusation, condamnation, sacrifice: la Description de la Louisiane annoncerait-elle en filigrane l'histoire même du récollet, sacrifié à des intérêts dont il ne mesure sans doute pas alors lui-même la portée ${ }^{34}$ ?

Le lecteur sentira lui aussi les contrecoups de cette « répugnance» par deux fois associée, dans le récit, au statut religieux du narrateur. En effet, de ce voyage de plus de «deux cent lieues par eau» depuis Crèvecoeur, avant la rencontre des Sioux, la Description de la Louisiane ne précise à aucun moment l'orientation ${ }^{35}$. Simple oubli? Il s'agit d'autre chose. Manifestement, le narrateur ne parvient pas à crever l'abcès qui le ronge et préfère noyer son voyage dans une alternative indécidable:

32. Nouvelle Découverte, 238-239. C'est moi qui souligne.

33. Description de la Louisiane, 211.

34. La terminologie familiale employée par Hennepin lors de son séjour chez les Sioux et qui fait du religieux, par la bouche de l'Amérindien, tantôt un Père, un Fils ou un Esprit, pourrait aller également dans le sens allégorique du sacrifice d'une personne au service d'une collectivité.

35. Avant moi, J. G. Shea avait déjà souligné ce silence sur la direction prise par le missionnaire: «A question still remains as to what he really did do on leaving Fort Crevecoeur. In his first work, as we have already remarked, he states that he was sent to explore the Illinois to its mouth, or to visit some tribes where a mission was to be established; and he tells us that he had some design of going down the Mississippi to the gulf, but he nowhere says that he ascended it before he was taken.»Op. cit., 105. 
Nous avions consideré le Fleuve Colbert avec beaucoup de plaisir, \& sans aucun obstacle pour sçavoir s'il estoit navigable haut $\&$ bas $^{36}$.

Nous avions quelque dessein de nous rendre jusques à l'emboucheure du Fleuve Colbert [...] mais ces Nations qui se saisirent de nous, ne nous donnerent pas le temps de naviger haut $\&$ bas de ce Fleuve ${ }^{37}$.

La comparaison de la Description de la Louisiane avec la Relation des Découvertes qui lui a vraisemblablement servi de canevas ${ }^{38}$ confirme que cette ambiguïté réitérée pourrait n'être pas le fait du hasard, mais procéderait bien d'une intention. Par exemple, alors que la $R e$ lation des Découvertes donne pour probable la direction du Mississippi en aval de la rivière Illinois, la Description de la Louisiane est plus catégorique quant à cette direction: si la Relation des Découvertes dit clairement au lecteur que le sud du Mississippi n'a pas encore été exploré, la modification apparemment anodine du même passage dans la Description de la Louisiane efface cette certitude et permet à l'imagination de prêter au narrateur une connaissance beaucoup plus étendue du territoire.

La riviere Mississippi au dessous de la Divine paroit aller au sud sudouest, et au dessus elle vient du nord et du nord nordouest ${ }^{39}$.

Le Fleuve Colbert va au Sud-Sud'Oüest, \& vient du Nord \& du Nord'Oüest ${ }^{40}$.

Cette modification a des répercussions sur la description du Mississippi. Ainsi, l'énumération des affluents du grand fleuve n'est pas clairement restreinte, dans la Description de la Louisiane, à l'espace officiellement exploré entre l'embouchure de la rivière Illinois, au sud, et la chute appelée aujourd'hui Saint-Anthony, au nord ${ }^{41}$.

36. Description de la Louisiane, 206.

37. Ibid., 218.

38. A. Louant, loc. cit., 63-76; C. Broué, «Édition critique de la Relation des Découvertes », communication présentée au $65^{\mathrm{e}}$ congrès de l'ACFAS, Université du Québec à TroisRivières, 1997; C. Broué, Édition critique de la Description de la Louisiane et de la Nouvelle Découverte, op. cit., 25-34.

39. Relation des Découvertes, f. $23 \mathrm{r}^{\circ}$. C'est moi qui souligne.

40. Description de la Louisiane, 194. C'est moi qui souligne.

41. Libérée de cette ambiguïté géographique, la Nouvelle Découverte rétablira d'ailleurs la précision que le lecteur attend d'un récit de voyage: «Il ne reçoit aucune Riviere considerable du côté de l'Oüest depuis l'embouchure de la Riviere des Illinois jusques au Saut de St. Antoine de Padoüe, excepté celle des Otenta, \& une autre qui vient de l'Oûest Nord-Oüest à sept ou lieües de ce Saut. » Nouvelle Découverte, 313. C'est moi qui souligne. 
Le Fleuve Colbert va au Sud-Sud'Oüest, \& vient du Nord \& du Nord'Oüest [...]. Il ne reçoit aucune Riviere considerable du costé de l'Oüest, que celle des Otontenta, \& une autre qui vient du Oüest Nord'Oüest à sept à huit lieuës du Sault Saint Antoine de Pade $^{42}$.

Au sortir de la rivière Illinois, le canot bifurque-t-il à droite ou à gauche sur le Mississippi ? Le lecteur ne pourra en décider qu'en vertu d'un formidable acte de foi !

Pas davantage de renseignements ne nous sont fournis quant à ces nations éloignées dont le groupe aurait eu connaissance par Amérindiens interposés. Certes, la Description de la Louisiane fait d'abord état de «quatre Nations, dont il est parlé dans la Relation du voyage de Ferdinand Soto dans la Floride qui sont celle de Tula, de Casquin, Cicaca \& Daminoia» qui vivraient, selon les Illinois, vers le sud aux abords du Mississippi ${ }^{43}$. Ailleurs, après avoir précisé à nouveau que les Peuples qu'il se proposait de visiter n'avaient jamais vu d'Européens, le narrateur rapporte également la visite de «quelques Sauvages [qui] arriverent de ces Nations éloignées au Village des Islinois », qui assurent le groupe de la navigabilité du Mississippi en aval de la rivière Illinois ${ }^{44}$. Cette navigabilité est encore confirmée par un jeune guerrier venant du $\operatorname{sud}^{45}$, puis par l'arrivée de plusieurs «Osages, Cicaca \& Akansa qui estoient venus du costé du Sud pour voir les François, \& pour achepter des Haches ${ }^{46}{ }^{\star}$. Sans rien affirmer, la Description de la Louisiane infléchit ainsi la lecture vers le sud du Mississippi. Pourtant, ces nations mentionnées correspondent sans doute davantage au voyage proposé par le père Hennepin qu'à celui qui lui autait été imposé. Certains indices incitent en effet à croire que le voyage effectivement réalisé est d'une autre nature.

\section{DIRECTION OUEST?}

Bien qu'extrêmement parcimonieuses dans la Description de la Louisiane, certaines remarques de Hennepin confirmeraient que le

42. Description de la Louisiane, 196.

43. Description de la Louisiane, 150.

44. Ibid., 175-176.

45. Les renseignements précieux que fournit ce guerrier concernant les habitants et les affluents du Mississippi sont alors consignés par le missionnaire. C'est du moins ce qu'affirme la Description de la Louisiane, 177. On peut bien sûr mettre en doute le fait qu'Hennepin ait consigné cette information, ce geste étant attribué à La Salle dans la Relation des Découvertes. Qu'Hennepin plutôt que La Salle ait consigné les noms de nations est d'importance secondaire ici: le récit d'Hennepin signifie par là que les «nations ultérieures » que le missionnaire se proposait de visiter sont au sud.

46. Description de la Louisiane, 180. 
canot aurait suivi la rive ouest. Discrètement, le récit informe en effet qu'une fois à l'embouchure de la rivière Illinois, après avoir été arrêté quelques jours par les glaces, le canot traverse le fleuve:

nous continuâmes nostre route, traversans \& sondans de tous costés si le Fleuve estoit navigable; il est vray qu'il y a trois Islets au milieu prés de l'embouchure de la Riviere Seignelay, qui arrestent le bois ${ }^{47}[\ldots]$

Par ailleurs, la description du paysage à cette même embouchure sous-entend un regard tourné vers le couchant et révèle des visées colonisatrices pour ce territoire encore inexploré par les Européens:

À l'embouchure de la rivière Illinois: Du costé du Nord, vis à vis du Rocher, \& du côté de l'Oüest, au delà du Fleuve il y a des Campagnes de terre noire, dont on ne voit pas le bout, toutes prestes à estre cultivées, \& qui seroient tres-avantageuses pour la subsistance d'une Colonie ${ }^{48}$.

La correspondance de La Salle fait d'ailleurs état d'une appréciation semblable pour l'embouchure de la rivière des Illinois:

Elle [la grande rivière Mississippi ou la rivière des Islinois] baigne du costé du sud un rocher escarpé d'environ quarante pieds de haut propre à bastir un fort, et vis-à-vis elle arrose une belle prairie dont on ne voit point la fin, fort bonne à cultiver. Cette place me semble la plus convenable de toutes pour habiter, pour bien des raisons que je n'ay pas le loisir de déduire icy, et je pourray bien m'y establir au retour de mon voyage ${ }^{49}$.

La Nouvelle Découverte renforce cette impression de lecture en juxtaposant cet endroit idyllique où le regard se pose et la rivière «Otontenta ${ }^{50} »$ qui y coule.

entre les montagnes \& le Fleuve, il y a de grandes prairies, où on voit souvent paître des troupeaux de boeufs [...]. Au de là de cette montagne, on decouvre à perte de veüe de grandes Campagnes, que nous pouvons veritablement appeller les delices de l'Amerique. Dans cet endroit du côté de l'Oüest, il ne reçoit aucune Riviere considerable, que celle d'Otontenta, \& une autre, qui vient de l'Oüest Nord-Oüest à sept ou huit lieûes du Saut de S. Antoine de Padoüe, comme nous le verrons dans la suite ${ }^{51}$.

47. Description de la Louisiane, 194. C'est moi qui souligne.

48. Description de la Louisiane, 193.

49. P. Margry, op. cit., 248.

50. Qui pourrait correspondre à la rivière Des Moines, voir infra, note 67.

51. Nouvelle Découverte, 247-248. 
Assez curieusement, dans la Nouvelle Découverte, ce n'est d'ailleurs pas le sud du Mississippi qui attise l'enthousiasme du narrateur, mais justement ces «grandes prairies » qui s'étendent à perte de vue à l'ouest du grand fleuve et qui constituent, nous assure Hennepin, les «délices de l'Amérique». C'est également dans ce même ouvrage qu'un informateur, anonyme mais bien renseigné, prendra la forme d'un «On» pour affirmer que l'ouest est bien ce qu'il promet d'être :

on découvre les plus beaux pays du monde pendant la longueur de deux cens lieües. Nous ne pouvions nous lasser de les admirer. On nous a assuré, qu'en largeur ce sont de vastes Campagnes, où on trouve des terres admirables bordées de fois à autre par des côteaux extremement agreables, par des bois de haute fûtaie, \& par plusieurs bocages ${ }^{52}$.

Mais si le Paradis se dessine à l'ouest, il n'en demeure pas moins inaccessible au lecteur de la Description de la Louisiane. Et le silence obstiné du texte nous ramène encore à cette question: mais qu'allaitil faire dans ce canot?

\section{COMMERCE ILLICITE?}

Il apparaît indiscutable, compte tenu de la quantité de marchandises confiée aux trois hommes ${ }^{53}$, qu'un des objectifs de leur expédition était commercial. Un tel objectif s'accommode mal des déclarations ostensibles de désintéressement du père Hennepin prêchant pour son ordre, mais aussi des termes des lettres patentes accordées à La Salle, qui commandaient implicitement à l'explorateur de confiner ses activités au sud des Grands $\mathrm{Lacs}^{54}$, et a pu paraître suffisamment incriminant pour justifier, en 1682, le mutisme du récollet. On comprend moins bien, toutefois, qu'Hennepin ait gardé le même silence dans la Nouvelle Découverte, alors qu'il y déclare plus ouvertement son désaccord avec certains choix ou certains agissements de La Salle ${ }^{55}$. Par

52. Ibid., 295.

53. «Pour mil ou douze cens livres», Description de la Louisiane, 187; la marchandise transportée par un canot de deux hommes aurait permis de «faire plus de 4 à 5000 livres de pelleteries» d'après La Salle, dans P. Margry, op. cit., 67.

54. «Nous vous avons permis \& permettons par ces presentes [...] de travailler a la descouverte de la partie occidentalle de notre $\mathrm{d}[\mathrm{it}]$ pays de la nouvelle france [...] a condition neanmoins que [...] vous ne ferez aucun commerce avec les Sauvages appelez Outaouacs \& autres qui apportent leurs castors \& autres pelleteries a Montreal que vous ferez le tour a vos depens et ceux de votre compagnie», Permission au Sr de la Salle, f. 43 v $^{\circ}$. C'est moi qui souligne.

55. Une étude comparative des deux premiers ouvrages de Louis Hennepin révèle une certaine «mise en veilleuse» de l'explorateur au profit du protagoniste-narrateur. Actanciellement parlant, le personnage de La Salle, dont le statut prééminent dans la Description de 
ailleurs, la Description de la Louisiane nous informe que les hommes avaient convenu d'un rendez-vous avec La Salle à l'embouchure de la rivière Wisconsin ${ }^{56}$. Hennepin aurait-il été envoyé prêcher l'évangile aux peuples de la Baie des Puants? Le malaise du texte s'expliquerait alors, les jésuites ayant déjà fondé deux missions dans cette baie ${ }^{57}$. Toutefois, l'intention annoncée par Hennepin de s'établir parmi les peuples qu'il allait visiter ${ }^{58}$ et la formulation même de La Salle ${ }^{59}$, disant du récollet qu'il était allé prêcher l'évangile «à des peuples qui n'en avoient jamais ouy parler ${ }^{60} »$, semblent contredire cette hypothèse. Si, déjà en 1682, Hennepin brouille les pistes et ouvre son récit à un possible voyage vers le sud, la soif de gloire du récollet est-elle seule en cause? On pourrait le penser si l'examen de la correspondance de La Salle ne révélait pas le même embarras concernant le voyage des trois hommes.

\section{QU'EN DIT LA SALLE?}

Posons donc à nouveau la question en examinant la correspondance de La Salle: quelles sont les nations vivant en amont de la rivière Illinois, aux abords du Mississippi, chez qui le missionnaire Louis Hennepin accepte à contre-cœur de se rendre ? Ou, ce qui revient peut-être au même: à quel(s) peuple(s) amérindien(s) destinet-on les marchandises que les trois hommes emportent?

On trouve sous les explications enchevêtrées de La Salle quelques indices supplémentaires sur la mission de Louis Hennepin. En 1681,

la Louisiane en fait un protagoniste essentiel à la bonne marche de l'expédition, passerait plutôt du côté des opposants dans la Nouvelle Découverte. C. Broué, «Récit d'un voyage et voyage d'un récit. De la Description de la Louisiane à la Nouvelle Découverte du père Louis Hennepin », communication présentée au 47e congrès de l'IHAF, automne 1994.

56. Description de la Louisiane, 375. Ce rendez-vous est confirmé par La Salle dans sa lettre du 22 août 168[1] : «le R. P. Louis Hempin et Picard prirent résolution de venir jusqu'à l'emboucheure de la rivière où j'avois promis d'envoyer de mes nouvelles, comme j'avois fait par six hommes que les Jésuites desbauchèrent, en leur disant que le R. P. Louis et ses compagnons de voyage avoient esté tuez.» P. Margry, op. cit., 257.

57. L'ouverture des missions de Saint-Marc et de Saint-Jacques à l'est de la Baie des Puants remonte à 1669, d'après L. Kellogg, The French Regime in Wisconsin and the North West (New York, Cooper Square Publishers, 1968 [1925]), 160.

58. «Je m'offris à faire ce voyage pour tâcher d'aller faire connoissance avec les Peuples chez lesquels j'esperois bien tost m'établir pour prescher la Foy », Description de la Louisiane, 187.

59. «Je l'ay fait remonter [le Mississippi] par un canot conduit par deux de mes gens, l'un nommé Michel Accault et l'autre Picard, auxquels le R. P. Louis Hennepin se joignit pour ne perdre pas l'occasion de prescher l'Évangile aux peuples qui habitent dessus et qui n'en avoient jamais ouï parler», La Salle, dans P. Margry, op. cit., 245-246.

60. Lettre de La Salle du 29 septembre 1680, dans I. Paquet, op. cit., 173. 
voulant justifier l'envoi de ce canot et se disculper d'éventuelles accusations d'imprudence de sa part ${ }^{61}$, La Salle donne quelques renseignements sur les tribus qu'Hennepin se proposait d'évangéliser. Même s'il reste évasif quant à la destination finale des trois hommes, il explique que son intention n'avait pas été d'envoyer ces derniers chez les Sioux et mentionne certaines tribus chez qui ils auraient dû séjourner:

Je ne doute pas qu'on ne dise plusieurs choses de ce voyage: $1^{\circ}$ Que je devois envoyer un homme qui entendist la langue. A cela il est aisé de respondre que je n'envoyois pas Accault aux Nadouesioux, mais pour remonter la Grande Rivière; qu'il entendoit la langue de ceux qui estoient les plus proches, comme les Otoutanta, les Aiounouea, les Kikapou et Maskoutens Nadouesioux, chez qui il devoit passer auparavant, et de là prendre langue pour aller plus $\operatorname{loin}^{62}$.

Ces explications méritent qu'on s'y arrête. Les «Otoutanta» correspondraient aux Otos, des Sioux Chiweres ${ }^{63}$ dont un groupe est situé, sur la carte de Franquelin de 1688, à l'extrémité de la rivière Des Moines $^{64}$. Sur cette même carte, l'ethnonyme «Otoutenta» est utilisé concurremment avec celui de «Mascoutens Nadouessioux» (qui désigne, chez La Salle, les Sioux des plaines ${ }^{65}$ ). Il sert également à qualifier l'une des branches nord de la rivière Missouri66. Les "Ainoves " correspondraient à une autre tribu de Chiweres, les Iowas ${ }^{67}$, que les Relations des jésuites assimilent également aux Mascoutens Nadouessioux ${ }^{68}$. Otoutentas et Ainoves feraient donc partie de la même grande famille des «Maskoutens Nadouessioux» dont Michel Accault com-

61. Rappelons l'épître au roi de la Description de la Louisiane dans laquelle le père Louis rappelle la mort de son supérieur et affirme que lui-même a été, à l'occasion de ce voyage, «cruellement exposé ».

62. Lettre de La Salle du 22 août 168[1], dans P. Margry, op. cit., 258.

63. F. W. Hodge, Handbook of North American Indians (New York, Pageant Books, 1960), II : 164-165. L'édition originale date de 1907-1910.

64. Plusieurs chercheurs situent en effet ce groupe sur le bord de la rivière Des Moines dans les années 1680. R. G. Thwaites, dir., A New Discovery of a Vast Country in America by Father Louis Hennepin, I: 186 et II: 627; J. G. Shea, op. cit., 110. Néanmoins, il pourrait également s'agir de la rivière Iowa (voir J. G. Shea, ibid, et F. W. Hodge, op. cit., 165-166).

65. Y. Goddard, «Historical and Philological Evidence Regarding the Identification of the Mascouten », Ethnohistory, 19,2 (printemps 1972): 130, note 6.

66. J'ai consulté une reproduction de cette carte dans L. A. Brown, Early Maps of the Ohio Valley. A Selection of Maps, Plans and Views Made by Indians and Colonials from 1673 to 1783 (Pittsburg, University of Pittsburg Press, 1959), pl. 6.

67. F. W. Hodge, op. cit., $1: 612$.

68. R. G. Thwaites, The Jesuit Relations and Allied Documents. Travels and Explorations of the Jesuit Missionaries in New France, 1610-1791 (Cleveland, Burrows, 1896-1901), 60: 321, note 27. 
prenait la langue ${ }^{69}$ et chez qui lui et ses compagnons devaient séjourner avant d'aller «plus loin». Quant aux «Kikapous», culturellement et linguistiquement proches des Mascoutens ${ }^{70}$, ils étaient souvent associés à ces derniers ou aux Miamis ${ }^{71}$ dans les récits de la période dite historique $^{72}$. Franquelin les localise, en 1688, le long d'un affluent est du Mississippi, situé en amont par rapport à l'embouchure de la rivière Des Moines et en aval de la Wisconsin. Sur sa carte de 1697, Hennepin les situe au sud de la rivière Fox. Quoi qu'il en soit, la formulation choisie par La Salle suggère qu'en 1680, des Chiweres et des Kikapous vivaient ensemble ou à proximité les uns des autres quelque part sur le trajet que devaient emprunter les trois hommes.

Par ailleurs, dans une lettre précédente, La Salle avait signalé, parmi les Amérindiens venus chez les Illinois pour rencontrer les Français, la nation des «Matoutentas» et celle des «Chaa ${ }^{73} »$. Ces derniers les auraient invités à aller chez eux, et c'est à ce voyage qu'Hennepin se serait offert de prendre part.

ils [les engagés] furent encores plus enco(u)ragés quand ils virent arriver des Cicaças des Akansa et des Osages qui aya(nt) appris nostre arrivée estoient venus du costé du sud pour voir des François et forger des haches [...] d'un autre costé, le 17e fevrier deux des plus notables de la nation des Matoutenta qui demeurent à 80 ou 100 lieues vers le couch(ant), arriverent pour nous voir [...] d'autres appelés Chaa qui demeurent au haut de la grande riviere arriverent le $24 \mathrm{e}$ fevrier et nous invitere(nt) à aller chez eux où ils disent avoir grande quantité de castor et de pelleterie et estre voysins de la mer de l'ouest un des troix reverends Peres Recollect(s) qui m'avoient accompagné jusqu'alors accompagné [sic] de deux de mes plus braves hommes s'offrit à faire ce voyage pour ne pas perdre cette occasion d'annoncer l'Evangile à des peuples qui n'en avoient jamais ouy parler ${ }^{74}$.

69. F. W. Hodge met en doute cette affirmation de La Salle à l'effet que Michel Accault connaissait la langue de ces peuples (op. cit., I: 613). Toutefois, La Salle affirme qu'Accault savait parler la langue de «ceux qui étaient les plus proches, comme les Otoutanta, les Aiounouea, les Kikapou et Maskoutens Nadouessioux ». Lettre de La Salle du 22 août 168[1] dans P. Margry, op. cit., 258.

70. C. Callender, «Kickapoo», dans B. Trigger, Northeast Handbook of North American Indians (Washington, Smithsonian Institution), 15: 656-667. Voir notamment R. G. Thwaites, The Jesuit Relations, 59 (1675): 220, sur la mission Saint-Jacques.

71. Cette fois, il s'agit plutôt d'un groupe algonquin semi-sédentaire dont les Relations des Jésuites font également mention sous l'appellation de «Nation du feu». Sur cette tribu, voir l'article de Y. Goddard, loc. cit., 123-132.

72. C. Callender, «Miami», dans B. Trigger, op. cit., 681-689.

73. Lettre du 29 septembre 1680, dans I. Paquet, op. cit., 171-173.

74. Ibid., 168-171. 
Les maigres indications géographiques de La Salle nous permettent de situer ces Matoutentas à l'ouest du Mississippi, à environ $400 \mathrm{~km}$ du fort Crèvecœur. Or, d'après ce qu'écrit La Salle lui-même des affluents ouest du Mississippi en amont de la rivière Illinois, les tribus «Otoutantas Paoté et Maskoutens, Nadouesioux » seraient disposées de part et d'autre d'une rivière ${ }^{75}$ dont l'embouchure serait située à environ $400 \mathrm{~km}$ de la rivière Illinois.

Le Mississippi ne reçoit point de rivières considérables du costé de l'ouest, depuis la rivière des Islinois jusqu'au pays des Nadouesioux, que celle des Otoutantas Paoté et Maskoutens, Nadouesioux du costé du levant, et environ cent lieües de Téakiki $^{76}$.

Cette similitude des données géographiques porte à croire que «Matoutenta» et «Otoutenta» correspondent, sous la plume de La Salle, à une seule et même tribu. Quant à l'ethnonyme Chaa, il correspond sans doute à l'appellation contemporaine de Cheyennes, un peuple de langue algonquienne dont on dit qu'ils viennent du sud des Grands Lacs et s'installent dans le Dakota du Sud à la fin du XVII siècle $^{77}$. N. H. Winchell situe cette tribu sur les bords de la rivière Minnesota autour des années $1680^{78}$. Selon La Salle, ces Cheyennes « disent avoir grande quantité de castor et de pelleterie ${ }^{79} »$. Voilà, sans doute, la destination de ces marchandises qui alourdissent le canot.

Hennepin et les deux engagés de La Salle doivent-ils passer chez les deux tribus mentionnées par La Salle ou seulement chez les Cheyennes? La lettre de 1680 n'en dit pas plus. Rapprochée de celle de 1681, elle permet toutefois d'avancer que le voyage des trois hommes comportait deux grandes étapes chez des groupes situés à l'ouest du Mississippi : celui des Chiweres (Ottos et Iowa, apparemment associés à des Kikapous) sur un affluent ouest situé au sud de la rivière Wisconsin et celui des Cheyennes, vivant vraisemblablement sur la Minnesota, auxquels le gros des marchandises semblait destiné.

75. C'est du moins ce que je comprends de sa description pour le moins confuse du seul affluent ouest qu'il dit juger valable.

76. La Salle, 22 août 168[1], dans P. Margry, op. cit., 249.

77. F. W. Hodge, op. cit., I: 250-251.

78. Sur une carte intitulée «Distribution of the Dakota in Minnesota at the Time of Hennepin Based on History and Tradition», N. H. Winchell situe les Cheyennes (SHIEN) à l'ouest des Iowas sur la rivière Minnesota. The Aborigines of Minnesota: A Report Based on the Collections of Jacob C. Brower, and on the Field Surveys of Alfred J. Hill and Theodore H. Lewis (Saint Paul, Minnesota, The Pionneer Co., 1911), 68.

79. Lettre de La Salle dans I. Paquet, op. cit., 172. 


\section{UNE RELECTURE DES OUVRAGES D'HENNEPIN S'IMPOSE}

Riches de ces quelques renseignements supplémentaires, revenons aux ouvrages d'Hennepin. La Description de la Louisiane mentionne la «rivière des Otontentas», présentée comme l'un des deux seuls affluents importants à l'ouest du Mississippi. Le deuxième affluent n'est pas nommé, mais peut correspondre à la rivière Minnesota.

Dans la Nouvelle Découverte, rien à première vue ne semble ajouter à cette description. Pourtant, la disparité entre le texte et la carte qui l'accompagne se trouverait expliquée par cette hypothèse d'une incursion vers l'ouest (voir la carte). Ainsi, le Missouri qui, d'après le texte, prendrait sa source dans une montagne, correspond sur la carte à la «Riviere Otenta» :

Ces peuples (les Issatis), dont je savois la langue, par ce que j'eus occasion de l'apprendre pendant le sejour, que je fis ensuite parmi eux, m'ont appris, que cette Riviere des Osages, \& de Messorites étoit formée de quantité d'autres, \& qu'on en trouve la Source en remontant à dix ou douze journées de Chemin à une montagne, d'où on voit sortir tous ces ruisseaux, qui composent ensuite cette Riviere. Ils ajoutoient, qu'au de là de cette montagne on voit la Mer, \& de grands Vaisseaux ${ }^{80}$.

Le narrateur aurait-il transformé des notes prises lors de son voyage vers l'ouest pour qu'elles s'intègrent à sa description d'une descente imaginée du Mississippi ? Ou aurait-il plutôt adapté une carte tracée par d'autres pour rendre compte de son propre voyage? Dans cette perspective, un tout nouveau sens se dégagerait de ce passage où Hennepin affirme qu'il serait en mesure de trouver un passage «commode» pour se rendre en Chine et au Japon: «Ceux qui auront leu ma Relation, \& qui examineront un peu les Cartes, qu'on y a jointes, reconnoîtront aisément la verité de ce que je dis ${ }^{81}$.» Le récollet aurait laissé au lecteur attentif toutes les clefs nécessaires pour accéder à la «vérité » de son voyage! 217.

80. Nouvelle Découverte, 253. Un passage similaire se trouve dans C. Leclercq, op. cit.,

81. Ibid., 373. Cette description se lit comme suit dans C. Leclercq: «Il y a de plus les peuples Miamis scituez au Sud quard de Sudest du fond du Lac Dauphin sur le bord d'une assez belle riviere environ quinze lieuës dans les terres à quarante un degré de latitude Septentrionale. La nation des Maskoutens \& des Outagamys qui demeurent environ à 43. degrez de latitude sur le bord de la riviere appellée Melleoki qui se décharge assez prés de leur Village dans le Lac Dauphin: du costé de l'Oüest les Kikapous, \& les Ainoves qui ont deux Villages, à l'Oüest de ces derniers au haut de la riviere de Checagoumemant. Le Village des Illinois Cascaschia scitué à l'Oüest du fond du Lac Dauphin tirant un peu au Sudouest environ les 41. degrez de latitude Les Anthoutantas, \& Maskoutens, Nadouessions environ 130. lieuës des Illinois dans trois 


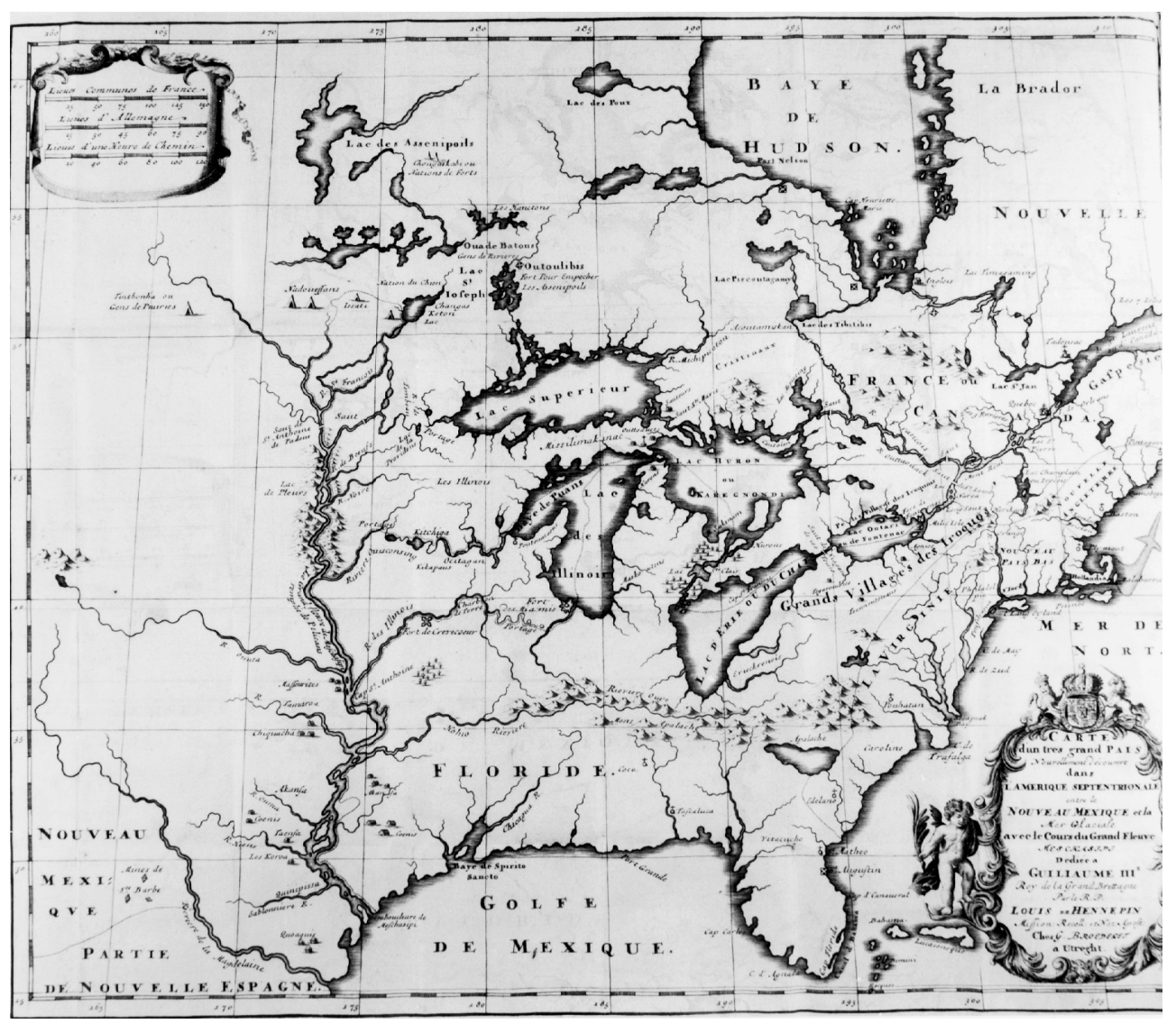

Carte d'un tres grand pays entre le Nouveau Mexique et la Mer Glaciale, dans Nouvelle Decouverte d'un Tres grand Pays Situé dans l'Amérique, 1697, gravure extraite de l'exemplaire de la Bibliothèque nationale, RES AF 95. 
Qu'apprend-on de plus à la lecture de la Nouvelle Découverte? Une description de différentes tribus Miamis situées à l'ouest du lac Michigan, description qui ne se trouvait pas dans la Description de la Louisiane, a particulièrement attiré mon attention.

Il est bon d'observer ici, qu'il y a des Miamis situez au SudOüest du fond du Lac des Illinois. Ils habitent sur le bord d'une Riviere assez belle, qui est environ à quinze lieües dans les terres à quarante \& un degré de latitude Septentrionale. La Nation des Maskoutens \& celle des Outoüagamis demeurent environ à quarante trois degrez de latitude sur le bord de la Riviere appelée Melleoki, qui se décharge assez prés de leur Village dans le Lac des Illinois. Du côté de l'Oüest on trouve les Kikapous, \& les Ainoves, qui ont deux Villages. A l'Oüest de ces derniers au haut de la Riviere de Chécagoumenant il y a un autre Village d'Illinois Cascaschia situé à l'Oüest du fond du même Lac, tirant un peu à Sud-Oüest environ les 41. degrez de latitude. Les Authoutantas, \& Maskoutens Nadoüessiouz demeurent à cent trente lieües des Illinois dans trois grands Villages bâtis proche d'une Riviere, qui se décharge dans le Fleuve Meschasipi. C'est du côté de l'Oüiest au dessus de la Riviere des Illinois vis à vis de l'embouchure de Oüisconsin, il y a une autre Riviere, qui se décharge dans le même Fleuve ${ }^{82}$.

L'inventaire des tribus, présenté au début d'un chapitre dont l'intitulé annonce la mauvaise disposition des Illinois à la religion chrétienne ${ }^{83}$, semble détaché de la narration et ne s'intégrer à aucun des événements qui ponctuent la chronologie du voyage. Pourtant, plusieurs détails (distances, latitude, nombre de villages, appréciation des sites) donnent à penser que le narrateur a une connaissance précise de certains des villages qu'il inventorie, dont ceux des Authoutentas et des Maskoutens Nadouessioux. On peut ainsi considérer cette description comme un élément de subversion du récit: sa présence même contredit ce que le narrateur lui-même raconte de l'expédition puis-

grands Villages bâtis proche d'une riviere qui se décharge dans le Fleuve Colbert du costé de l'Oüest au dessus de celle des Illinois quasi vis-à-vis l'embouchure de Miskoncing dans le même Fleuve, je pourrois encore citer icy quantité d'autres Nations, où nous communiquons \& où les François coureurs de bois, où legitimement envoyez se répandoient dans le temps que j'étois aux Illinois à la faveur de nostre découverte.»Op. cit., 173-174. La parenté de certains passages de la Nouvelle Découverte et du Premier établissement de la Foy est indéniable, mais le débat sur la paternité des deux ouvres est loin d'être réglé. Aussi ne m'apparaît-il pas vain de scruter ces passages revendiqués par Hennepin.

82. Nouvelle Découverte, 218-219. C'est moi qui souligne.

83. Chapitre XXXIII, «Reflexion sur l'humeur des Illinois, avec un petit détail du peu de fruit, qu'on pouvoit ésperer de leur conversion», Nouvelle Découverte, 217. 
qu'elle suppose une connaissance du territoire qui n'est pas étayée d'une présence déclarée du missionnaire sur les lieux.

Dans cet inventaire, le lecteur apprend que des Kikapous et des Iowas vivent à proximité les uns des autres quelque part à l'ouest du lac Michigan. «Authoutantas» et «Maskoutens Nadouessiouz» constituent par ailleurs un groupement distinct que l'auteur situe à 130 lieues des Illinois sur le bord d'un affluent du Mississippi. Quel est cet affluent? Malgré une formulation là encore approximative, on comprend qu'il s'agit d'un affluent ouest qui se trouve en amont de la rivière Illinois ${ }^{84}$. Cette «autre Riviere, qui se décharge dans le même Fleuve» pourrait être la rivière Des Moines ou encore l'Iowa.

Par sa singularité, ce passage pourrait confirmer ce que la correspondance de La Salle nous laisse entrevoir: les trois hommes auraient exploré un ou des affluents ouest du Mississippi avant d'être surpris par les Dakotas sur le bord du grand Fleuve. Il pourrait s'agir de la rivière Des Moines: les distances données par le récollet retrouveraient alors une cohérence qu'elles ne peuvent avoir quand on n'envisage qu'une navigation restreinte au seul Mississippi ${ }^{85}$.

\section{DES MOTIFS INAVOUABLES}

Mais si véritablement l'exploration de l'ouest faisait partie de la mission des trois hommes, pourquoi avoir déployé tant d'efforts pour la tenir dans l'ombre? La renommée d'Hennepin autant que celle de La Salle n'auraient-elles pas profité de ces «découvertes ${ }^{86} »$ ? Plusieurs explications me viennent à l'esprit: un échec des trois hommes pourrait expliquer que personne n'ait cherché à revendiquer une quelconque avancée territoriale. La Salle lui-même aurait pu vouloir garder secrètes ses tentatives d'exploration vers l'ouest, soit pour ne pas

84. La formulation utilisée dans C. Leclercq, op. cit., nuance ce «vis à vis de l'embouchure» par un «quasi vis-à-vis» sans doute géographiquement plus juste, voir supra, note 81 .

85. Ainsi, les calculs auxquels s'est livré J. Delanglez sur la valeur de la lieue chez Hennepin - qui lui servent à prouver que le récollet s'est servi de données fournies par un autre explorateur ou d'autres commerçants et aurait été incapable d'une quelconque cohérence s'appuient, entre autres, sur cette indication donnée par la Description de la Louisiane : «Nous avions fait environ deux cens lieux par eau, depuis nostre depart des Islinois, \& nous navigâmes avec ces Sauvages qui nous prirent pendant dix-neuf jours, tantost au Nord, tantost au Nord'Oüest [...] depuis ce temps-là nous fimes environ deux cens cinquante lieuës de chemin sur le Fleuve Colbert, mesme davantage.» Description de la Louisiane, 219. C'est moi qui souligne. Toutefois, J. Delanglez considère qu'Hennepin ne parle que de la remontée du Mississippi, alors que la formulation choisie par le récollet reste ouverte à d'autres eaux que celle du grand fleuve. Voir Hennepin's Description of Louisiana, op. cit. (1941), 95-96.

86. Estimant que si La Salle avait eu connaissance d'un passage vers l'ouest, il en aurait nécessairement parlé, J. Delanglez refuse d'envisager que l'explorateur ait pu essayer de trouver la mer de l'ouest. «A Mirage: The Sea of the West», loc. cit., 366-369. 
déplaire au roi, soit pour éviter de se faire «damer le pion» par un autre explorateur ou d'autres commerçants. Pourtant alors, comment expliquer que la Nouvelle Découverte persiste, après la mort de La Salle, à masquer cette partie de son voyage en proclamant non pas la découverte de l'ouest, mais celle de l'embouchure du Mississippi? Tenter de répondre à cette question fait surgir d'autres surprises du texte d'Hennepin.

Curieusement en effet, la rencontre d'Hennepin et de ses compagnons avec des Sioux soulage le missionnaire, malgré l'inquiétude que le comportement inusité de ces Amérindiens suscite chez lui: "ainsi leur ayant fait rompre leur voyage, je n'estois pas fâché dans cette conjecture de continuer nos Decouvertes avec ces Peuples ${ }^{87}$.» Incidemment, elle révèle au lecteur un nouvel indice quant à ce qui a précédé cette rencontre: interrogés par les Sioux, les trois hommes leur apprennent que les Miamis, que les Sioux recherchent, «avoient pris la fuite, et traversez le Fleuve Colbert pour se joindre aux Islinois ${ }^{88} »$. Il semblerait en effet que des guerriers illinois avaient fait incursion en territoire sioux pendant l'hiver et que des combats avaient fait plusieurs morts de part et d'autre ${ }^{89}$.

D'où les trois hommes tiennent-ils leur information? Il se peut que cette rencontre des Miamis avec les Illinois ait eu lieu avant leur départ de Crèvecœur, ce qui expliquerait que le missionnaire soit au courant des allées et venues de la tribu miami ${ }^{90}$. Toutefois, la correspondance de La Salle suggère une seconde hypothèse: les trois hommes auraient été eux-mêmes partie prenante de ce déplacement des «Miamis» vers le pays des Illinois.

La Salle, racontant le voyage du récollet et des deux hommes, rapporte en effet de son côté qu' "une bande de cent guerriers Nadouesioux, qui venoient à dessein de tuer des Tchatchakigoua descendoit le mesme fleuve dans trente-trois canots d'escorce de bouleau ${ }^{91} . » \mathrm{Au}$ début de sa lettre, La Salle avait expliqué que les Tchatchakigoa $^{92}$ constituaient la tribu principale de la nation Miami,

87. Description de la Louisiane, 212.

88. Ibid., 208-209. La direction des Sioux est claire: ils «descendaient d'une vitesse extraordinaire, pour aller en guerre aux Miamis, aux Islinois \& Maroha», 207. C'est moi qui souligne.

89. C. Leclercq, op. cit., 205.

90. L'alliance Illinois-Miami est corroborée par la Description de la Louisiane qui mentionne que «les Miamis vinrent [au mois de février] danser le Calumet aux Islinois», 181

91. Lettre de La Salle du 22 août 168[1], dans P. Margry, op. cit., 255.

92. S'agirait-il du groupe miami des Atchachakangouen dont C. Callender dit qu'ils formaient l'une des six tribus regroupées collectivement sous l'appellation de Miamis? Voir «Miami», dans B. G. Trigger, op. cit., 681. 
établie «à l'ouest du lac des Islinois, en deçà de la Grande Rivière », qui «s'estoient enfuis au delà de la rivière Colbert, vers le Couchant, parmy les Otoutanta, les Paoté et les Maskoutens, Nadouessioux, qui les avoient receus par force depuis quatre ans ${ }^{93}$.» Les jésuites auraient eu des contacts pendant plusieurs années avec ces Amérindiens qu'ils auraient, selon La Salle, essayé de faire déménager au printemps de 1679 en favorisant un rapprochement avec les Aoiatenon, Maskoutens [Nadouessioux ?], Kalatekoë ${ }^{94}$ et Pepepoaké ${ }^{95}$, et leur installation entre les Iroquois et les Illinois, "pour faire alliance avec les Iroquois et faire avec eux la guerre aux Islinois ${ }^{96} »$. Associés d'abord aux Maskoutens $^{97}$, ces Miamis s'en seraient dissociés, si l'on en croit La Salle, à la suite de l'échec d'une tentative d'alliance avec les Tsonnontouans: on aurait fait croire aux députés des deux tribus qu'ils risquaient la mort en restant au village iroquois.

une femme venue du village de Gannagaro, où demeuroit le Père Raffeix, de qui je l'ay tousjours creue envoyée [...] coucha avec un d'eux, et luy dit en secret que le lendemain on leur devoit casser la teste, et qu'ils n'avoient point d'autre party à prendre que celuy de la fuite [...] En effect, ceux-cy prirent la fuite à l'heure mesme, à la réserve d'un qui estoit couché dans une autre cabane [...] L'un d'eux, s'estant caché dans un arbre creux, y fust descouvert par ses pistes, qui y conduisirent des Tsonnontouans qui tombèrent dessus et l'emmenèrent au village, où il dit le motif de leur départ. [...] Cette affaire en demeura là jusqu'à mon départ, la défiance qu'on avoit inspirée aux Iroquois de moy et de mon voyage rendant inutiles toutes mes recherches et tous les biais que je pris pour en descouvrir le secret. Tout ce que j'appris, ce fut qu'il s'agissoit de nouer une forte intelligence entre les Iroquois et les Miamis, pour ruiner les Islinois ${ }^{98}$.

Des Maskoutens auraient par la suite tenté de faire échouer l'installation de La Salle dans le pays illinois en y députant un de leurs

93. Lettre de La Salle du 22 août 168[1], dans P. Margry, op. cit., 215.

94. L'un des examinateurs de la Revue suggère qu'il pourrait s'agir du groupe des Kilatikas, une autre tribu miami. C. Callender, loc. cit., 681.

95. Lettre de La Salle du 22 août 168[1], dans P. Margry, op. cit., 216. Les Pepikokia constituaient une tribu ou une bande algonquienne considérée, au XVII ${ }^{\mathrm{e}}$ siècle, comme une branche de la nation Miami (C. Callender, loc. cit., 681), et sont signalés au début du XVIII ${ }^{e}$ comme un village de Wea (Oiatinons). F. W. Hodge, op. cit., 228-229.

96. Voyage de M. de La Salle à la rivière Mississippi, dans P. Margry, Découvertes et établissements des Français, II : 99.

97. Cette fois des Miamis Maskoutens, différents des Maskoutens Nadouessioux.

98. P. Margry, op. cit., 219. 
chefs, Monso, qui aurait affirmé que La Salle était de connivence avec les Iroquois ${ }^{99}$.

dès que la nouvelle a esté portée au village où est le Père d'Allouez que j'estois arrivé aux Islinois, on a député le nommé Monso, un des chefs, qui a apporté sous terre quatre grandes chaudières, douze haches et vingt couteaux, pour dire aux Islinois que $\mathrm{j}$ 'estois frère de l'Iroquois, que je respirois son haleine, que je mangeois les serpens de son pays, qu'ils m'avoient donné une seine pour les envelopper d'un costé, pendant que les Iroquois venoient de l'autre, que j'estois hay de toutes les Robes Noires qui m'abandonnoient, ne me regardant que comme un Iroquois; que $\mathrm{j}$ 'avois voulu desjà tuer les Miamis, que $\mathrm{j}$ 'en avois pris deux prisonniers, et que j'avois de la médecine pour empoisonner tout le monde ${ }^{100}$.

Le même épisode, dans la Description de la Louisiane, donne justement lieu à une autre incohérence du récit, cette fois chronologique. Hennepin impute aux menées de ce chef le départ de six hommes, qui se sont déjà pourtant enfuis d'après son propre récit ${ }^{101}$. Les divergences entre la version de La Salle et celle du récollet suggèrent en outre qu'une certaine mauvaise foi entoure cette affaire:

Ce discours [de la Salle] les émut \& les obligea à faire courir après Monso pour le ramener, mais la neige qui tomba la nuit en abondance, \& qui couvroit les pistes empescha qu'on ne le pût joindre ${ }^{102}$.

Il me fut aisé de destruire toutes ces faussetez, et peu s'en fallut que ce pauvre Monso n'y demeurast pour les gages, luy ayant esté respondu que c'estoit luy qui avoit le serpent Iroquois sous la langue, que ses camarades qui y avoient esté en ambassade en avoient apporté et n'avoient pu fumer dans le mesme calumet sans respirer les haleines Iroquoises. - Si je ne m'estois opposé, les Islinois auroient tué ce Monso ${ }^{103}$.

Enfin, la Description de la Louisiane affirme, par la bouche de Monso, que La Salle et sa troupe ont bien l'intention d'étendre leur

99. Les lettres de La Salle et la Description de la Louisiane disent seulement de Monso qu'il est un chef miami. Néanmoins, la Nouvelle Découverte précise qu'il est Maskoutens, et qu'il avait été choisi pour cette ambassade «parce que les Illinois avoient plus de créance en lui qu'aux autres Miamis » (p. 206).

100. Voyage de M. de La Salle à la rivière Mississippi, dans P. Margry, op. cit., 100 .

101. Description de la Louisiane, 154 et 162.

102. Ibid., 161.

103. Voyage de M. de La Salle à la rivière Mississippi dans P. Margry, op. cit., 100. 
commerce ou leur influence à l'ouest du Mississippi au détriment des Illinois :

il assembla secretement les Anciens, \& les asseura que nous voulions aller joindre leurs ennemis qui demeurent au delà de la grande Riviere Colbert, que nous leurs fournirions des armes \& des munitions, \& qu'aprés les avoir assemblez nous nous joindrions aux Iroquois \& les enfermerions de tous costez pour les exterminez entierement ${ }^{104}$.

Récapitulons. Au début de l'année 1680, des Tchachakigoas, Miamis alliés des Iowas ${ }^{105}$, résidant sur l'un des affluents ouest du Mississippi, viennent tout juste de s'installer entre les Iroquois et les Illinois, exactement sur le passage de La Salle, après avoir entrepris des négociations avec les Tsonnontouans depuis l'été de 1679, dans le village desquels La Salle lui-même se trouvait alors de passage. Dans les tout premiers jours de janvier 1680, des Maskoutens, qui refusent de s'allier avec les Iroquois, tentent de persuader les Illinois que La Salle cherche leur perte et tente pour cela de s'associer les tribus environnantes ${ }^{106}$. La Salle soutient au contraire que les jésuites (nommément le père Allouez) tentent de lui barrer la route en favorisant une entente entre Iroquois et Miamis, contre les Illinois. À la fin de février, pourtant, l'explorateur envoie trois hommes, dont un missionnaire récollet, chez les Iowas, alliés des Tchatchakigoa dont le lecteur de la Description de la Louisiane apprend le déménagement vers le pays des Illinois au début d'avril. Revenu en France, le récollet garde un silence farouche sur le contact (ou la tentative de contact) qu'il aurait établi avec ces nations ou leurs alliés, tout en manifestant sa «répugnance» pour un voyage semblant mettre en jeu son honneur.

Qu'allait-il donc faire dans cette galère? Louis Hennepin auraitil trempé dans une sordide machination visant à faire place nette à $\mathrm{La}$ Salle, déterminé à s'installer dans le pays des Illinois et, par la même

104. Description de la Louisiane, 152. La lettre de La Salle ne souffle mot de cette précision.

105. Ces Tchachakigoas correspondraient à cette tribu de Miamis que La Salle affirme s'être déplacée sur les instances réitérées des jésuites.

106. Selon Y. Goddard, il se serait produit, en 1679, une scission entre les Mascoutens et les Miamis qui aurait conduit à la division des Mascoutens en deux groupes: un premier groupe associé généralement aux Miamis, à l'extrémité sud du lac Michigan, et un groupe situé plus à l'ouest associé aux Renards et aux Kikapous le long des rivières Fox et Wisconsin et vers le haut Mississippi, loc. cit., 127. Y. Goddard, qui s'appuie sur la Relation des Découvertes reproduite dans P. Margry, op. cit., I: 463 et sur la lettre de La Salle du 22 août 168[1] dans P. Margry, II : 216-220, remarque que cette scission expliquerait la dispersion géographique et la variété des associations politiques qu'auraient contractées les Mascoutens jusqu'au début du $\mathrm{XVIII}^{\mathrm{e}}$ siècle. 
occasion, à salire la réputation de la Compagnie de Jésus? Une lettre de J. Dudouyt à Laval semblerait accréditer cette hypothèse:

le pere louis [...] avoit seize lettres des peres jesuistes du Canada quil faisoit voir et publioit par tout et pretendoit convaincre par ces lettres les Jesuistes de beaucoup de choses et entre autre, quils faisoient la traitte, qu'ils divertissoient le castor par la Hollande: et que cetoient eux quy avoient fait faire la guerre par les iroquois aux illinois que cetoit pour enveloper $\mathrm{mr}$ de la Salle et ses gens avec eux. Il sest fondé sur une lettre du p. dablon quil pretendoit estre une preuve convaincante pour faire voir que les Jesuistes avoient fait faire cette guerre aux illinois par les iroquois ${ }^{107}$.

Une telle entorse à sa condition ecclésiastique expliquerait à merveille les nombreuses contradictions qui parsèment l'ensemble de l'œuvre du récollet et peut-être, aussi, son bannissement du territoire français par ses pairs, quelques années plus tard. Cette interprétation de la Description de la Louisiane autoriserait en outre le réexamen de la Nouvelle Découverte et de l'épisode de la descente du Mississippi et conférerait un sens nouveau à des remarques attribuables, au premier abord, à la «forfanterie» du récollet.

Ils [les pères Gabriel de la Ribourde, Luc Buisset, Zenobe Membré et Mélithon Watteau] me firent connoître, que j'avois beaucoup souffert dans ma Mission pendant l'hyver [...]. Je dissimulay une partie, de ce qui s'étoit passé, par ce que je voulois attirer avec moy les Peres Gabriel, \& Zenobe dans nôtre Découverte. D'ailleurs je savois, que le Sieur de la Salle, qui étoit alors au Fort de Frontenac, \& dont je connoissois la conduite par experience, se servoit volontiers de cette fameuse maxime, Divide \& impera, \& qu'ils souhaitoit de l'insinuer entre ses gens pour en disposer plus aisément selon ses desseins ${ }^{108}$.

La publication de ce deuxième ouvrage de Louis Hennepin s'expliquerait alors non plus comme une bravade de la part de l'ancien missionnaire, mais plutôt comme une tentative de réhabilitation auprès de ses supérieurs ${ }^{109}$. Le réexamen de tous les ouvrages du père Hennepin à la lumière de cette hypothèse pourrait ainsi révéler certains «trous noirs» des agissements de La Salle.

107. Mars 1682, ASQ, carton N, $\mathrm{n}^{\circ} 61$, reproduite par H. Lemay, $\mathrm{n}^{\circ} 26,17$.

108. Nouvelle Découverte, 106-107.

109. Alors que la Description de la Louisiane est traversée par de multiples polémiques opposant l'ordre de Saint-François à la Compagnie de Jésus et à l'évêque de Québec, la Nouvelle Découverte insiste à plusieurs reprises sur la solidarité de Louis Hennepin avec les missionnaires jésuites de la Nouvelle-France. Voir, entre autres, 114-155. 
En somme, malgré la réputation peu enviable réservée par l'histoire aux textes du récollet Louis Hennepin, la Description de la Louisiane pourrait ouvrir un nouveau point de vue aux recherches sur La Salle et sur les dynamiques intertribales en Nouvelle-France, entre 1678 et 1681. Le relevé des contradictions ou des ellipses du texte permet de reconstituer un passé noyé sous des enchevêtrements de demi-vérités, d'embrouilles et de silences : le récollet et ses deux compagnons auraient été chargés d'explorer le territoire à l'ouest du Mississippi. Une comparaison avec les écrits de La Salle suggère que les trois hommes devaient d'abord passer chez les Iowas, sur la rivière Des Moines ou un autre affluent ouest en aval de l'embouchure de la Wisconsin, avant de poursuivre leur remontée du Mississippi jusqu'à la rivière Minnesota, chez les Cheyennes. Si cette dernière étape semble motivée par le commerce, il n'en est pas de même de la première, et la rencontre inopinée du groupe avec des Sioux n'aurait pas empêché le bouillant Hennepin d'être mêlé à une intrigue menée par le célèbre explorateur visant à allier Iroquois, Miamis et Iowas contre les Illinois afin d'expulser ceux-ci de leur territoire, et à imputer la responsabilité de ce conflit aux missionnaires jésuites. Malgré la fragilité de cette hypothèse combinant le non-dit du texte de la Description de la Louisiane, les assertions de la correspondance de La Salle et quelques ajouts de la Nouvelle Découverte, la lecture nouvelle qui en résulte pourrait ouvrir à la recherche sur La Salle et l'exploration du Mississippi de nouvelles perspectives. 